Olivia Sgarbura*, Clarisse Eveno, Mohammad Alyami, Naoual Bakrin, Delia Cortes Guiral, Wim Ceelen, Xavier Delgadillo, Thanh Dellinger, Andrea Di Giorgio, Amaniel Kefleyesus, Vladimir Khomiakov, Michael Bau Mortensen, Jamie Murphy, Marc Pocard, Marc Reymond, Manuela Robella, Koen P. Rovers, Jimmy So, S.P. Somashekhar, Clemens Tempfer, Kurt Van der Speeten, Laurent Villeneuve, Wei Peng Yong and Martin Hübner

\title{
Consensus statement for treatment protocols in pressurized intraperitoneal aerosol chemotherapy (PIPAC)
}

https://doi.org/10.1515/pp-2022-0102

Received January 10, 2022; accepted February 2, 2022;

published online March 1, 2022

\begin{abstract}
Objectives: Safe implementation and thorough evaluation of new treatments require prospective data monitoring and standardization of treatments. Pressurized intraperitoneal
\end{abstract}

*Corresponding author: Olivia Sgarbura, Department of Surgical Oncology, Cancer Institute of Montpellier, University of Montpellier, Montpellier, France; and IRCM, Institut de Recherche en Cancérologie de Montpellier, INSERM U1194, Université de Montpellier, Institut régional du Cancer de Montpellier, Montpellier, France,

E-mail: Olivia.Sgarbura@icm.unicancer.fr. https://orcid.org/00000002-6965-3697

Clarisse Eveno, Department of Digestive and Oncological Surgery, University of Lille, Claude Huriez University Hospital, Lille,

France

Mohammad Alyami and Delia Cortes Guiral, Department of General Surgery and Surgical Oncology, Oncology Center, King Khalid Hospital, Najran, Saudi Arabia. https://orcid.org/0000-0002-38342271 (D.C. Guiral)

Naoual Bakrin, Department of General Surgery \& Surgical Oncology, Centre Hospitalier Lyon-Sud, Hospices Civils

de Lyon, Pierre-Bénite, France; and Lyon University 1, EA 3738 CICLY, Lyon, France

Wim Ceelen, Department of GI Surgery, Ghent University Hospital, Ghent, Belgium. https://orcid.org/0000-0001-7692-4419

Xavier Delgadillo, Centre Médico Chirurgical Volta, Unité Spécialisée de Chirurgie, La Chaux-de-Fonds, Switzerland. https://orcid.org/ 0000-0003-4236-6310

Thanh Dellinger, Department of Gynecologic Oncology, City of Hope National Medical Center, Duarte, CA, USA

Andrea Di Giorgio, Peritoneal and Retroperitoneal Surgical Unit, Fondazione Policlinico Universitario Agostino Gemelli IRCCS, Rome, Italy. https://orcid.org/0000-0003-3981-1145

Amaniel Kefleyesus, Department of General Surgery \& Surgical Oncology, Centre Hospitalier Lyon-Sud, Hospices Civils de Lyon, Pierre-Bénite, France; and Department of Visceral Surgery, Lausanne University Hospital CHUV, University of Lausanne (UNIL), Lausanne, Switzerland, E-mail: amaniel.kefleyesus@chuv.ch. https://orcid.org/0000-0002-7951-4041 aerosol chemotherapy (PIPAC) is a promising alternative for the treatment of patients with peritoneal disease with an increasing number of suggested drug regimens. The aim was to reach expert consensus on current PIPAC treatment protocols and to define the most important research topics.

Methods: The expert panel included the most active PIPAC centers, organizers of PIPAC courses and principal investigators of prospective studies on PIPAC. A comprehensive

Vladimir Khomiakov, P.A. Hertsen Moscow Research Oncological Institute - Branch of the National Medical Research Center of Radiology, Moscow, Russia

Michael Bau Mortensen, Department of Surgery, Odense Pancreas Center (OPAC) \& Odense PIPAC Center (OPC), Odense University Hospital, Odense, Denmark. https://orcid.org/0000-0002-7270-5005 Jamie Murphy, Academic Surgical Unit, Imperial College Healthcare NHS Trust, London, UK

Marc Pocard, Université de Paris, INSERM, U1275 CAP Paris-Tech, Paris, France; and Hepato-Biliary-Pancreatic Gastrointestinal Surgery and Liver Transplantation, Pitié Salpêtrière Hospital, AP-HP, Paris, France

Marc Reymond, Department of Surgery, University of Tübingen, Tübingen, Germany

Manuela Robella, Unit of Surgical Oncology, Candiolo Cancer Institute-FPO, IRCCS, Turin, Italy

Koen P. Rovers, Department of Surgery, Catharina Cancer Institute, Eindhoven, The Netherlands

Jimmy So, Division of Surgical Oncology, National University Cancer Institute, Singapore, Singapore

S.P. Somashekhar, Department of Surgical Oncology, Manipal Comprehensive Cancer Center, Manipal Hospital, Bangalore, India Clemens Tempfer, Department of Obstetrics and Gynecology and Therapy Center for Peritoneal Carcinomatosis, Marien Hospital Herne, Ruhr-Universität Bochum, Herne, Germany

Kurt Van der Speeten, Department of Surgical Oncology, Ziekenhuis Oost-Limburg, Genk, Belgium

Laurent Villeneuve, Lyon University 1, EA 3738 CICLY, Lyon, France; and Department of Public Health, Clinical Research and Epidemiology, Hospices Civils de Lyon, Lyon, France

Wei Peng Yong, Cancer Science Institute of Singapore, National University of Singapore, Singapore, Singapore

Martin Hübner, Department of Visceral Surgery, Lausanne University Hospital CHUV, University of Lausanne (UNIL), Lausanne, Switzerland 
literature review served as base for a two-day hybrid consensus meeting which was accompanied by a modified three-round Delphi process. Consensus bar was set at $70 \%$ for combined (strong and weak) positive or negative votes according to GRADE. Research questions were prioritized from 0 to 10 (highest importance).

Results: Twenty-two out of 26 invited experts completed the entire consensus process. Consensus was reached for 10/10 final questions. The combination of doxorubicin $\left(2.1 \mathrm{mg} / \mathrm{m}^{2}\right)$ and cisplatin $\left(10.5 \mathrm{mg} / \mathrm{m}^{2}\right)$ was endorsed by $20 /$ 22 experts (90.9\%). 16/22 (72.7\%) supported oxaliplatin at 120 with potential reduction to $90 \mathrm{mg} / \mathrm{m}^{2}$ (frail patients), and $77.2 \%$ suggested PIPAC-Ox in combination with 5-FU. Mitomycin-C and Nab-paclitaxel were favoured as alternative regimens. The most important research questions concerned PIPAC conditions $(n=3)$, standard $(n=4)$ and alternative regimens $(n=5)$ and efficacy of PIPAC treatment $(n=2) ; 8 / 14$ were given a priority of $\geq 8 / 10$.

Conclusions: The current consensus should help to limit heterogeneity of treatment protocols but underlines the utmost importance of further research.

Keywords: cisplatin; doxorubicin; oxaliplatin; peritoneal metastasis; PIPAC; standardisation; treatment protocol.

\section{Background}

Pressurized intraperitoneal aerosol chemotherapy (PIPAC) has been proposed in 2011 as novel modality for the palliative treatment of peritoneal metastases (PM) of various origins. Two empirical protocols have been suggested by the pioneer group, namely (I) oxaliplatin (Ox) at $92 \mathrm{mg} / \mathrm{m}^{2}$ body surface i.e. $20 \%$ of the Elias regimen for hyperthermic intraperitoneal chemotherapy (HIPEC) for PM of colorectal and appendicular origin and (II) the combination treatment of doxorubicin (D) $1.5 \mathrm{mg} / \mathrm{m}^{2}$ and cisplatin (C) 7.5 $\mathrm{mg} / \mathrm{m}^{2}$ for all other entities representing each $\leq 10 \%$ of current HIPEC doses [1-3] (Table 1). Feasibility, safety and excellent tolerance has been demonstrated repeatedly along with promising clinical outcomes, essentially by the use of these two drug regimens [4-6].

A first dose escalation study for PIPAC-D/C was terminated without dose-limiting toxicity (DLT) and without reaching the maximally tolerated dose (MTD) suggesting an increase by 35\% (2.1/10.5) [7]. Most active PIPAC centers adopted this "new" dose without formal validation by a phase II study [8], and the new dose was also accepted for the ISSPP PIPAC course curriculum [9].

Two dose-escalation studies for PIPAC-Ox have been conducted in the same time period in Nantes and Singapore. The French study was terminated at a dose of 90 due to DLTs, while the Singapore study was terminated with the final escalation dose of 120, again without reaching the MTD $[10,11]$.

The most recent Italian study suggested significantly higher doses without DLTs and without reaching the MTD, namely 135 for PIPAC-Ox and 6/30 for PIPAC-D/C, respectively [12]. However, this study included only a single-shot PIPAC and was terminated prematurely due to administrative limitations. Of note, PIPAC was administered as monotherapy in most of these studies, while in current practice $72 \%$ of patients receive PIPAC as add-on treatment embedded in cycles of systemic chemotherapy [8]. Therefore, tolerance and cumulative toxicity might be different for patients under combination therapy and careful consideration is needed before accepting dose increase of the well-established drug regimens. Furthermore, several teams started to add concomitant 5-FU during PIPAC-Ox administration [13]. However, this did not jeopardize tolerance and safety profile in a multicenter retrospective cohort of patients who received ePIPAC in majority combined with systemic chemotherapy [14]. Table 2 provides a comparative overview for the pivotal studies on the different PIPAC-Ox regimens [10-12].

Table 1: Overview on drug regimens used for PIPAC, HIPEC, and systemic treatment.

\begin{tabular}{|c|c|c|c|c|c|c|}
\hline & PIPAC dose, $\mathrm{mg} / \mathrm{m}^{2}$ & IV dose, $\mathrm{mg} / \mathrm{m}^{2}$ & HIPEC dose, $\mathrm{mg} / \mathrm{m}^{2}$ & NIPS dose, $\mathrm{mg} / \mathrm{m}^{2}$ & PIPAC/IV \% & PIPAC/HIPEC, \% \\
\hline $0 x$ & $46-135$ & 85 & $200-460$ & $85-130$ & $50-160$ & $10-30$ \\
\hline Dox & $1.5-2.1$ & 15 & $15^{\mathrm{a}}$ & NA & $10-13$ & 10 \\
\hline Cis & $7.5-10.5$ & 75 & $50^{\mathrm{a}}$ & NA & $10-13$ & $15-21$ \\
\hline MMC & $1.5(14 \mathrm{mg} \text { total dose })^{\mathrm{c}}$ & 20 & $10-35$ & NA & $43-75$ & $4-15$ \\
\hline Iri & $20^{c}$ & $125-180$ & 200 & NA & $11-16$ & $10(0.1)$ \\
\hline Ptx & $30^{b}$ & $135-175$ & $60-175$ & $20-80$ & $17-22$ & $17-50$ \\
\hline Nab-Ptx & 112,5 & 125 & NA & NA & 90 & NA \\
\hline
\end{tabular}

${ }^{\mathrm{a}}$ Doses only used for the combination of drugs, not for the independent use of each drug; ${ }^{\mathrm{b}}$ dose based only on animal studies; ${ }^{\mathrm{c}}$ doses based on expert opinion and not published in the literature. 
Table 2: Comparison of PIPAC-Ox regimens and their dose-finding studies.

\begin{tabular}{|c|c|c|c|}
\hline RP2D & $\begin{array}{l}\text { PIPOX study } \\
90 \mathrm{mg} / \mathrm{m}^{2}\end{array}$ & $\begin{array}{l}\text { NUH study } \\
120 \mathrm{mg} / \mathrm{m}^{2}\end{array}$ & $\begin{array}{l}\text { Turin study } \\
135 \mathrm{mg} / \mathrm{m}^{2}\end{array}$ \\
\hline Study design & $3+3$ & $3+3$ & Continual reassessment method \\
\hline DLT defined & $\begin{array}{l}\text { Any grade III or IV toxicity or unexpected } \\
\text { post-operative complication. }\end{array}$ & Any grade 3 toxicity & Not defined \\
\hline Predefined dose levels & $90,145,200,255,300$ & $45,60,90,120$ & $100,135,155$ \\
\hline No of included patients & 10 & 17 & 6 \\
\hline Repeated PIPAC & 10 & 8 & No \\
\hline $\mathrm{sCT}$ & Yes & No & No \\
\hline Grade 3 toxicity & $\begin{array}{l}\text { Nausea, neutropenia, anemia, } \\
\text { hypersensitivity to } \mathrm{Pt} \text {, hemorrhage, } \\
\text { obstruction }\end{array}$ & $\begin{array}{l}\text { Acute pancreatitis in } \\
\text { the first dose level }\end{array}$ & No \\
\hline Response at P2RD & PRGS3 & PRGS1 & NR \\
\hline Origin of PM & Gastric, CRC + App & Gastric, CRC + App, HPB & Gastric, CRC + App, HPB \\
\hline \multirow[t]{2}{*}{ Criticism } & $\begin{array}{l}\text { Hypersensitivity considered as a DLT } \\
\text { while it is not dose-dependent }\end{array}$ & DLT not attained & DLT not clearly defined \\
\hline & $\begin{array}{l}\text { Neutropenia usually excluded from } \\
\text { other studies or used as a combined parameter. }\end{array}$ & $\begin{array}{l}\text { Last level not doubled } \\
(n=3)\end{array}$ & $\begin{array}{l}\text { Very limited number of included patients } \\
\text { Last dose level not doubled } \\
\text { (inclusions stopped because of } \\
\text { insurance issues) }\end{array}$ \\
\hline
\end{tabular}

App, appendiceal cancer; CRC, colorectal cancer; CRM, continual reassessment method; DLT, dose limiting toxicity; HPB, hepato-pancreatobiliary malignancies; NR, not reported; P2RD, phase 2 recommended dose; PRGS, peritoneal regression grading score; SCT, systemic chemotherapy.

Next, new indications for PIPAC treatment have been recently proposed beyond a purely palliative approach. For a neoadjuvant approach with potentially curative approach (secondary cytoreductive surgery \pm HIPEC), maximization of treatment effect is the highest priority and higher doses and hence high risk for adverse events (AE) appear acceptable. However, high rate of AEs is inacceptable for PIPAC with prophylactic/adjuvant intention in a setting of unknown benefit for any intraperitoneal chemotherapy [15-17]. The Odense team proposed therefore to lower ox dose by 50\% (46) in their prospective study on prophylactic PIPAC in colorectal cancer patients with high risk for development of PM (NCT03280511) [17].

Finally, other drugs (Nab-paclitaxel for ex.) and drug combinations (Nab-paclitaxel + cis) are under evaluation (NCT03304210, NCT04000906) [18], while others (mitomycin-c (MMC), irinotecan (IRI)) are already used in clinical practice in case of intolerance, allergy etc. Little has been published on these drugs, and different empirical doses are discussed via social media [19].

The aim of this process was to scrutinize currently practiced treatment protocols for PIPAC to reach expert consensus for clinical practice and to identify the most important research questions.

\section{Methods}

The consensus process followed current recommendations and was previously applied to standardize safety and technical aspects of PIPAC treatment [20-24]:

(1) Expert panel: eligible were members of the ISSPP education committee with large personal experience with PIPAC treatment and the principal investigators of prospective studies on PIPAC. The core group (OS, CE, and $\mathrm{MH}$ ) contacted all eligible persons three times at least and no colleague was deliberately excluded. Requirements for participation were participation in the hybrid consensus meeting and the three-round Delphi process.

(2) The core group prepared a comprehensive overview on PIPAC regimens to prepare the consensus meeting including the best available evidence and important confidential information from key opinion leaders.

(3) The consensus meeting (Supplementary Material, Appendix 1) was held on July 2nd and 3rd 2021 in Paris and virtual participation (via zoom) was granted according to sanitary requirements due to the COVID-19 pandemic. The program included expert lectures on methodology of dose finding, pharmacology, PIPAC technology and individual presentation of all dose-finding studies by the respective PIs. Controversial aspects were discussed and important research questions were defined. The protocol of the meeting served as basis to prepare the final voting.

(4) A modified Delphi approach was used to reach final consensus: The core group prepared a first inquiry based on the evidence. All participants were required to answer the online survey (Survey Monkey, San Mateo, CA, USA, www.momentive.ai) before the 
consensus meeting. Feedback was provided in the first part of the consensus meeting and a modified version of the survey was repeated live with instantaneous feedback (AhaSlides, AhaSlides Pte Ltd, Singapore, ahaslides.com) at the end of the meeting. The core group prepared the final Delphi round based on the discussions and conclusions during the consensus meeting (Supplementary Material, Appendix 2). This final round was sent again to all panelists via Survey Monkey and every participant had four weeks for completion and received four reminders at least.

(5) Presentation, discussion and planned validation of the results of the consensus process were planned during the ISSPP bi-annual meeting in Rome, October 7-8th.

The requirements for consensus were an overall response rate higher than $70 \%$ and a unidirectional recommendation of more than $70 \%$.

The statistical analysis was mainly descriptive based on the data provided by the web-based platforms (Survey Monkey, AhaSlides). The graphics were prepared by use of Excel software (Microsoft Corp., Redmond, WA, USA).

\section{Results}

Overall, 26 experts were eligible and were invited to participate. Twenty two of them participated in all steps of the consensus process. Nineteen participants were surgical oncologists, two gynecologists and one medical oncologist. 2/22 had additional expertise in pharmacology.

Final Delphi three questions (Supplementary Material, Appendix 2) were phrased after expert presentations and in-depth discussion during the two-day hybrid meeting (Supplementary Material, Appendix 1). The panel shared full agreement $(100 \%)$ for the impact of other variables than drug regimens for PIPAC, endorsement of general principles to define dosing in oncology, but also for the importance of standardization of PIPAC regimens (Figure 1).

There was a strong agreement in favor of uniform PIPAC regimens for the different indications and tumor entities. The combination of doxorubicin $\left(2.1 \mathrm{mg} / \mathrm{m}^{2}\right)$ and cisplatin $\left(10.5 \mathrm{mg} / \mathrm{m}^{2}\right)$ was endorsed by $20 / 22$ experts (90.9\%). 16/22 (72.7\%) supported oxaliplatin at 120 with potential reduction to $90 \mathrm{mg} / \mathrm{m}^{2}$ (frail patients), and $77.2 \%$ suggested PIPAC-Ox in combination with 5-FU (Figure 1). Mitomycin-C and Nab-paclitaxel were favoured as alternative regimens. Suggested dosing for the different drugs are provided as online Supplementary Material, Appendix 3.

The most important research questions concerned PIPAC conditions $(n=3)$, standard $(n=4)$, and alternative regimens $(n=5)$ and efficacy of PIPAC treatment $(n=2)$; eight out of 14 topics were rated to have a priority of $\geq 8 / 10$ (Figure 2). Of note 10/14 topics were related to drugs and drug regimens.

\section{Discussion}

The expert panel reached consensus on PIPAC regimens for clinical practice while waiting for the results of ongoing

\begin{abstract}
Different other variables than the applied drug(s) (e.g. pressure, duration, temperature electrostatic precipitation, etc.) have an important impact on efficacy and side effects.

Defining the optimal dose for PIPAC treatment has to follow the same methodological principles as for other systemic or locoregional chemotherapy treatments.
\end{abstract}

In absence of phase II studies, an expert consensus on the currently used regimens is useful in order to maintain safe and efficacious treatments, limit heterogeneity, and provide guidance for new centers.

In the lack of more data, uniform drug regimens should be used for PIPAC-Ox for the different indications (palliative, neoadjuvant, adjuvant/prophylactic) and settings (monotherapy, bidirectional).

PIPAC-Ox should be combined with intravenous 5-FU

The recommended dose for PIPAC-Ox for routine clinical use, PIPAC course material and outside clinical trials should be $120 \mathrm{mg} / \mathrm{m} 2$ with possible dose reduction to $90 \mathrm{mg} / \mathrm{m} 2$ (frail patients, neuropathy, combined treatment, etc.).

In the lack of more data, uniform drug regimens should be used for PIPAC-DC (doxorubicin/cisplatin) for the different indications (palliative, neoadjuvant, adjuvant/prophylactic) and settings (monotherapy, bi-directional).

The recommended dose for PIPAC-DC for routine clinical use, PIPAC course material and outside clinical trials should be $2.1 / 10.5 \mathrm{mg} / \mathrm{m} 2$

Evidence for PIPAC using other drugs than Ox or DC is insufficientand alternative drug (regimens) cannotbe recommended at this point of time for routine clinical use.

PIPAC with alternative drug regimens can be considered by a multidisciplinary tumor board a compassionate use in patients with no reasonable treatmentalternative and contraindication for PIPAC- OX/PIPAC-DC.

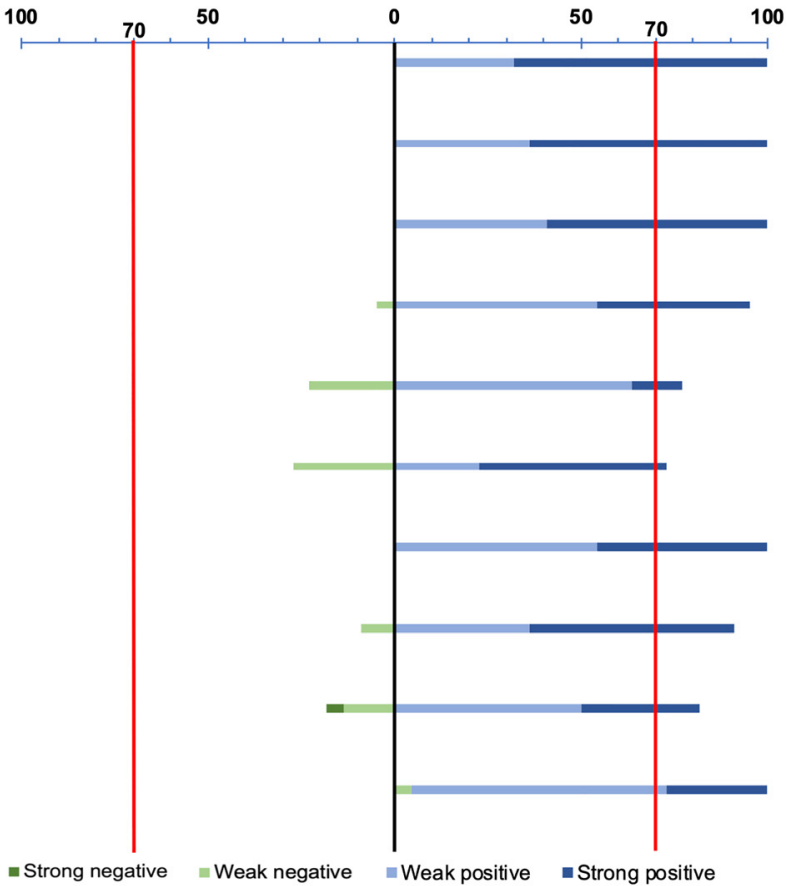

Figure 1: Expert consensus on PIPAC regimens. 
Preclinical studies to optimize conditions for ePIPAC.

Clinical studies to compare efficacy and safety of PIPAC vs EPIPAC.

Preclinical studies to optimize the other variables of PIPAC treatment including temperature, pressure, duration, carrier solution.

Another dose-escalation study for PIPAC-Ox aiming to increase the dose and hence efficacy.

Phase II study for PIPAC-Ox in order to validate one of the doses of the phase-I studies.

Another dose-escalation study for PIPAC-DC aiming to increase the dose and hence efficacy.

Phase Il study for PIPAC-DC in order to validate the dose of 2.1/10.5 $\mathrm{mg} / \mathrm{m} 2$.

Exploring alternative PIPAC regimens20. Phase II study for PIPAC-

Phase I study to define the optimal dose for Mitomycin-C.

Phase I study to define the optimal dose for Irinotecan.

Phase I study to define the optimal dose for Paclitaxel.

Phase I studies for other drugs or drug combinations.

Efficacy of PIPAC treatment25. Phase ll study to compare PIPAC-Ox with or without iv. 5-FU.

Phase III studies by tumor entity.
Pannelists research priorities

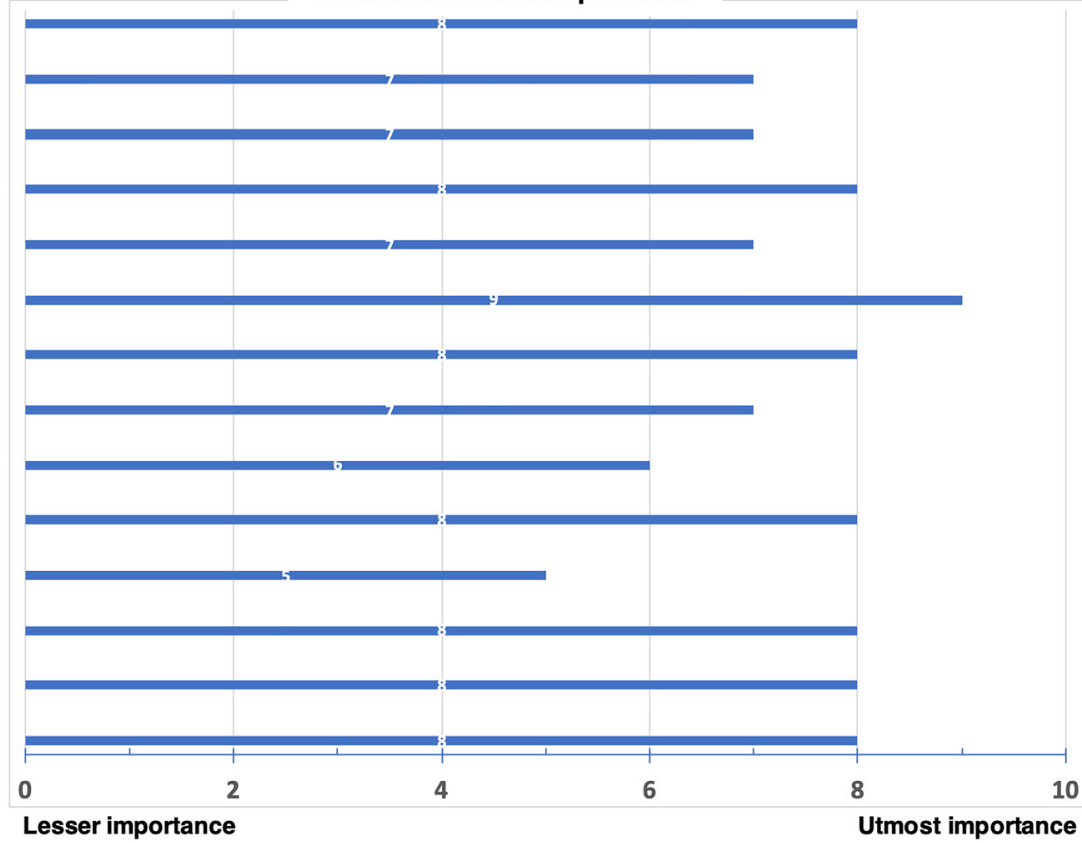

Figure 2: Research priorities to optimize PIPAC treatment.

The most important research questions were identified and discussed during the consensus meeting. Panelists rated importance of the topics on a scale from 0 to 10 (highest importance).

studies. Compared to the initial empirical regimens, higher doses were suggested based on the available phase-I studies and concrete alternatives were proposed in case of contraindication. There is high need for further research and the consensus panel identified the most important topics.

The development of PIPAC technology has followed until now the IDEAL stepwise process [25, 26]. Given the number of reported cases and centers [8], it can be considered that an early majority of adopters are now confronted with the diverging results of several phase I trials and they may find difficult to either change the regimen based on conflicting new data or keep the classical regimens they were trained to administer [9]. In order to avoid heterogeneity in the upcoming IDEAL $2 b$ studies and a plethora of PIPAC regimens similar to that of HIPEC regimens [27], it belongs to the experts to compare and synthetize current data in order to select recommended regimens in the waiting of further research.

Phase I trials are a critical step for introducing translational knowledge into clinical practice by establishing the right dose and administration schedule before clinical assessment. They also evaluate the safety of the drug but give very little insight on efficacy [28]. In the traditional Rocket Model of drug development, phase II and III trials are needed in order to grant wide acceptance and integration of new regimens in clinical guidelines [29]. These two steps may require 4-8 years to complete, huge budgets and a waiting time that is often incompatible with the translational science development, resulting in already obsolete data [29, 30]. Rapid development of new regimens, especially in a setting in which there is no present standard treatment, may be based solely on efficacy Phase II trials [29]. That could be the case for PIPAC regimens given that they are often administered in patients with advanced disease that have no standard options of treatment and given that the safety of the procedure is high $[4,7,10-12]$. Nevertheless, the success of upcoming studies remains dependent on the selection of the best candidate regimen given the various results of the PIPAC phase I trials.

The expert panel analyzed the strengths and the limitations of the different available studies and proposed PIPAC Ox at $120 \mathrm{mg} / \mathrm{m}^{2}$ (except in frail patients where $90 \mathrm{mg} / \mathrm{m}^{2}$ should be administered) and PIPAC D/C at 2.1/ $10.5 \mathrm{mg} / \mathrm{m}^{2}$. However, new phase I studies and, in particular of PIPAC D/C where the DLT was not met and the study stopped at the highest proposed dose level, were highly recommended as research priorities. The expert group recommended the combination of PIPAC-Ox with 5-FU based on data about the association of the two drugs for HIPEC [13]. Safety and preliminary data for PIPAC-Ox and ePIPAC-Ox exists but the clinical advantage of the association remains to be proved [10, 14, 31]. 
While homogenous regimens are recommended, there is also a need for more drug options administered as PIPAC, given the large range of primaries responsible for the peritoneal metastases as well as the frequency of allergies to platins and neurotoxicity [8, 32-34]. Irinotecan and mitomycin $\mathrm{C}$ were identified as new priorities in the search of an alternative, particularly in the setting of colorectal cancer.

There is a high interest in ePIPAC that showed impressive distribution and penetration potential in translational studies [35, 36]. While associated morbidity was explored and remains similar to that of traditional PIPAC [14], some of the associated variables (time of exposure, activation of the generator) have still not gained consensus and need further research [23]. This prerogative was confirmed among the research priorities.

The current consensus emerged from a clinical need of PIPAC centers worldwide [8, 23]. In order to deliver recommendations, the expert panel was forced to take into account only current and, thus, limited evidence and to apply a modified Delphi methodology. This approach will probably assist clinicians and help revise the training modules on a medium term but will require itself a rapid updating.

As a consequence, these are neither formal recommendations nor guidelines and several limitations are acknowledged by the authors. The expert panel included mainly surgical oncologists with large PIPAC expertise. Nonetheless, gynecology, medical oncology, and pharmacology were also represented as reflected also by online Supplementary Material, Appendix 1. Due to limited and conflicting evidence, a pragmatic approach and modified Delphi were necessary to reach consensus. A revision of this consensus statement will be necessary as soon as new data is available.

In summary, this consensus aims to avoid diversification of PIPAC regimens to warrant a safe treatment and common standards allowing for prospective multicenter evaluation of its efficacy. Formal evaluation according to the usual standards in oncology need to be pursued and shall help to provide formal recommendations in the future.

\section{Research funding: None declared.}

Author contributions: All authors have accepted responsibility for the entire content of this manuscript and approved its submission.

Competing interests: The following disclosure were made: MH: 1. Research funding (institution): Nestlé health science. 2. Speaker honorary (institution): MSD, Nestlé, Fresenius, Capnomed, Encare. 3. Boards: ERAS society: chair education, ISSPP: chair education. MR: 1.Research funding: Capnomed GmbH, Fisher Paykel, Alesi Surgical. 2. Speaker honorary: Boerhinger Ingelheim. 3. Stock shareholder: Capnomed
GmbH. MP: Research funding: Capnomed GmbH, IDmed, INSERM laboratory unit. XD: Speaker honorary: Capnomed $\mathrm{GmbH}$, Consultant for Latin America. WPY: Speaker honorary: Astra Zeneca, BMS, Eli Lilly, Novartis. The rest of the authors have no competing interests.

Informed consent: Not applicable.

Ethical approval: Not applicable.

\section{References}

1. Solass W, Kerb R, Mürdter T, Giger-Pabst U, Strumberg D, Tempfer C, et al. Intraperitoneal chemotherapy of peritoneal carcinomatosis using pressurized aerosol as an alternative to liquid solution: first evidence for efficacy. Ann Surg Oncol 2014;21:553-9.

2. Reymond MA, Solass W, Giger-Pabst U, Strumberg D, Tannapfel A, Zieren J, et al. Efficacy and safety of pressurized intraperitoneal aerosol chemotherapy in women with recurrent gynaecological cancer and peritoneal carcinomatosis. Surg Endosc Other Intervent Tech 2014;28:54-191.

3. Ceelen WP, Flessner MF. Intraperitoneal therapy for peritoneal tumors: biophysics and clinical evidence. Nat Rev Clin Oncol 2010;7:108-15.

4. Alyami M, Hübner M, Grass F, Bakrin N, Villeneuve L, Laplace N, et al. Pressurised intraperitoneal aerosol chemotherapy: rationale, evidence, and potential indications. Lancet Oncol 2019;20:e368-77.

5. Winkler CS, Sandhu J, Pettke E, Merchea A, Fong Y, Kumara HMCS, et al. Pressurized intraperitoneal aerosol chemotherapy, a palliative treatment approach for patients with peritoneal carcinomatosis: description of method and systematic review of literature. Dis Colon Rectum 2020;63:242-55.

6. Hübner M, Grass F, Teixeira-Farinha H, Pache B, Mathevet $P$, Demartines N. Pressurized intraperitoneal aerosol chemotherapy practical aspects. Eur J Surg Oncol 2017;43:1102-9.

7. Tempfer CB, Giger-Pabst U, Seebacher V, Petersen M, Dogan A, Rezniczek GA. A phase I, single-arm, open-label, dose escalation study of intraperitoneal cisplatin and doxorubicin in patients with recurrent ovarian cancer and peritoneal carcinomatosis. Gynecol Oncol 2018;150:23-30.

8. Sgarbura O, Villeneuve L, Alyami M, Bakrin N, Torrent JJ, Eveno C, et al. Current practice of pressurized intraperitoneal aerosol chemotherapy (PIPAC): still standardized or on the verge of diversification? Eur J Surg Oncol 2021;47:149-56.

9. Alyami M, Sgarbura O, Khomyakov V, Horvath P, Vizzielli G, So J, et al. Standardizing training for pressurized intraperitoneal aerosol chemotherapy. Eur J Surg Oncol 2020;46:2270-5.

10. Dumont F, Passot C, Raoul JL, Kepenekian V, Lelièvre B, BoisdronCelle $M$, et al. A phase I dose-escalation study of oxaliplatin delivered via a laparoscopic approach using pressurised intraperitoneal aerosol chemotherapy for advanced peritoneal metastases of gastrointestinal tract cancers. Eur J Cancer 2020; 140:37-44.

11. Kim G, Tan HL, Sundar R, Lieske B, Chee CE, Ho J, et al. PIPAC-OX: a phase I study of oxaliplatin-based pressurized intraperitoneal aerosol chemotherapy in patients with peritoneal metastases. Clin Cancer Res 2020;27:1875-81. 
12. Robella M, De Simone M, Berchialla P, Argenziano M, Borsano A, Ansari $S$, et al. A phase I dose escalation study of oxaliplatin, cisplatin and doxorubicin applied as pipac in patients with peritoneal carcinomatosis. Cancers 2021;13:1-12.

13. Badrudin D, Sideris L, Leblond FA, Pichette V, Cloutier AS, Drolet $P$, et al. Rationale for the administration of systemic 5-FU in combination with heated intraperitonal oxaliplatin. Surg Oncol 2018;27:275-9.

14. Taibi A, Teixeira Farinha H, Durand Fontanier S, Sayedalamin Z, Hübner M, Sgarbura O. Pressurized intraperitoneal aerosol chemotherapy enhanced by electrostatic precipitation (ePIPAC) for patients with peritoneal metastases. Ann Surg Oncol 2021;28: 3852-60.

15. Goéré D, Glehen O, Quenet F, Guilloit J-M, Bereder J-M, Lorimier G, et al. Second-look surgery plus hyperthermic intraperitoneal chemotherapy vs. surveillance in patients at high risk of developing colorectal peritoneal metastases (PROPHYLOCHIPPRODIGE 15): a randomised, phase 3 study. Lancet Oncol 2020; 21:1147-54.

16. Klaver CEL, Wisselink DD, Punt CJA, Snaebjornsson P, Crezee J, Aalbers AGJ, et al. Adjuvant hyperthermic intraperitoneal chemotherapy in patients with locally advanced colon cancer (COLOPEC): a multicentre, open-label, randomised trial. Lancet Gastroenterol Hepatol 2019;4:761-70.

17. Graversen M, Detlefsen S, Fristrup C, Pfeiffer P, Mortensen MB. Adjuvant pressurized intraperitoneal aerosol chemotherapy (PIPAC) in resected high-risk colon cancer patients - study protocol for the PIPAC-OPC3 trial. A prospective, controlled phase 2 study. Pleura Peritoneum 2018;3:20180107.

18. Van De Sande L, Graversen M, Hubner M, Pocard M, Reymond M, Vaira $M$, et al. Intraperitoneal aerosolization of albuminstabilized paclitaxel nanoparticles (Abraxane ${ }^{T M}$ ) for peritoneal carcinomatosis - a phase I first-in-human study. Pleura Peritoneum 2018;3:20180112.

19. Grossman R, Sgarbura O, Hallet J, Søreide K. Social media in surgery: evolving role in research communication and beyond. Langenbeck's Arch Surg 2021;406:505-20.

20. Hübner M, Kusamura S, Villeneuve L, Al-Niaimi A, Alyami M, Balonov K, et al. Guidelines for perioperative care in cytoreductive surgery (CRS) with or without hyperthermic intraperitoneal chemotherapy (HIPEC): enhanced recovery after surgery $\left(\right.$ ERAS $\left.^{\circledR}\right)$ society recommendations-part I: preoperative and intraoperative management. Eur J Surg Oncol 2020;46: 2292-310.

21. Hübner M, Kusamura S, Villeneuve L, Al-Niaimi A, Alyami M, Balonov K, et al. Guidelines for perioperative care in cytoreductive surgery (CRS) with or without hyperthermic intraperitoneal chemotherapy (HIPEC): enhanced recovery after surgery $\left(\right.$ ERAS $\left.^{\circledR}\right)$ society recommendations-part II: postoperative management and special considerations. Eur J Surg Oncol 2020; 46:2311-23.

22. Clerc D, Hübner M, Ashwin KR, Somashekhar SP, Rau B, Ceelen W, et al. Current practice and perceptions of safety protocols for the use of intraperitoneal chemotherapy in the operating room: results of the IP-OR international survey. Pleura Peritoneum 2021;6:39-45.

23. Hübner M, Alyami M, Villeneuve L, Cortés-Guiral D, Nowacki M, So J, et al. Consensus guidelines for pressurized intraperitoneal aerosol chemotherapy: technical aspects and treatment protocols. Eur J Surg Oncol 2021. https://doi.org/10.1016/j.ejso. 2021.10.028.

24. Brindle M, Nelson G, Lobo DN, Ljungqvist O, Gustafsson UO. Recommendations from the ERAS ${ }^{\circledR}$ Society for standards for the development of enhanced recovery after surgery guidelines. BJS Open 2020;4:157-63.

25. McCulloch P, Altman DG, Campbell WB, Flum DR, Glasziou P, Marshall JC, et al. No surgical innovation without evaluation: the IDEAL recommendations. Lancet 2009;374:1105-12.

26. Tate SJ, Torkington J. Pressurized intraperitoneal aerosol chemotherapy: a review of the introduction of a new surgical technology using the IDEAL framework. BJS Open 2020;4:206-15.

27. Yurttas C, Hoffmann G, Tolios A, Haen SP, Schwab M, Königsrainer I, et al. Systematic review of variations in hyperthermic intraperitoneal chemotherapy (HIPEC) for peritoneal metastasis from colorectal cancer. J Clin Med 2018;7:567.

28. Weber JS, Levit LA, Adamson PC, Bruinooge S, Burris HA, Carducci MA, et al. American Society of Clinical Oncology policy statement update: the critical role of phase I trials in cancer research and treatment. J Clin Oncol 2015;33:278-84.

29. Verweij J, Hendriks HR, Zwierzina H, Hanauske, Wacheck V, Collignon 0 , et al. Innovation in oncology clinical trial design. Cancer Treat Rev 2019;74:15-20.

30. Taibi A, Sgarbura O, Hübner M. ASO author reflections: developing next-generation intraperitoneal chemotherapy. Ann Surg Oncol 2020;28:3861-2.

31. Rovers KP, Wassenaar ECE, Lurvink RJ, Creemers G-JM, Burger JWA, Los M, et al. Pressurized intraperitoneal aerosol chemotherapy (oxaliplatin) for unresectable colorectal peritoneal metastases: a multicenter, single-arm, phase II trial (CRC-PIPAC). Ann Surg Oncol 2021;28:5311-26.

32. Gauthier A, Mankouri F, Demoly P, Sgarbura O, Chiriac AM. Hypersensitivity reactions to platinum-based compounds in the context of pressurized intraperitoneal aerosol chemotherapy (PIPAC): description and management. Allergy Eur J Allergy Clin Immunol 2020;76:1882-5.

33. Sgarbura O, Hubner M, Alyami M, Eveno C, Gagnière J, Pache B, et al. Oxaliplatin use in pressurized intraperitoneal aerosole chemotherapy(PIPAC) is safe and well tolerated: a multicenter study. Eur J Surg Oncol 2019;45:e60.

34. Siebert M, Alyami M, Mercier F, Gallice C, Villeneuve L, Bérard F, et al. Severe hypersensitivity reactions to platinum compounds post-pressurized intraperitoneal aerosol chemotherapy (PIPAC): first literature report. Cancer Chemother Pharmacol 2019;83: 425-30.

35. Van de Sande L, Rahimi-Gorji M, Giordano S, Davoli E, Matteo C, Detlefsen $S$, et al. Electrostatic intraperitoneal aerosol delivery of nanoparticles: proof of concept and preclinical validation. Adv Healthc Mater 2020;9:1-11.

36. Willaert W, Van de Sande L, Van Daele E, Van De Putte D, Van Nieuwenhove $Y$, Pattyn P, et al. Safety and preliminary efficacy of electrostatic precipitation during pressurized intraperitoneal aerosol chemotherapy (PIPAC) for unresectable carcinomatosis. Eur J Surg Oncol 2019;45:2302-9.

Supplementary Material: The online version of this article offers supplementary material (https://doi.org/10.1515/pp-2022-0102). 